\title{
Structural Design and Kinematics Analysis of Steel Cutting Manipulator Xianghong $\mathrm{Xu}$

\author{
Shazhou Professional Institute of Technology, Zhangjiagang 215600, China \\ email: xuxianghong602@163.com
}

Keywords: Section steel; Cutting processes; Cutting manipulator; Forward and inverse kinematics

\begin{abstract}
Because of its superior mechanical properties and functions of good use, Steel is widely applied to building structures, bridges, vehicles, supporting frame, machinery and other various industries, and have a large amount of cutting. Due to the different cross-sectional shape and sizes of various steel, and shapes and needs to cutting stock vary, it has a very important practical significance to $\mathrm{CNC}$ cutting of some steel to meet the degree of automation and flexibility .In this paper, it studies the numerical control cutting craft to the common steel materials, on this basis, a Steel Cutting Manipulator equipment is designed that able to adapt to common steel and meet the needs of a variety of cutting processes. The forward and inverse kinematics mathematical model of the structure are established.
\end{abstract}

\section{Introduction}

Steel for its excellent mechanical properties and superior performance, is widely used in construction, marine engineering, petrochemical industry, automobile manufacturing and other industries, these industries are required to cut the amount of steel [1]. In order to cut the section steel to meet the relevant requirements of users, and in the cutting place to meet the welding process welding groove, the need for high efficiency and flexibility of the corresponding steel cutting equipment [2]. At present, the steel cutting machine on the market is mostly aimed at a special machine for profiled steel. It can not meet the requirements of small batch and many types of processing. Therefore, it is a strong demand to design and develop CNC steel cutting machine for machining multi - section steel. Based on the structural framework of four kinds of commonly used steel design, in order to better welding, it is necessary to cut the end of the shaped steel into a certain shape of groove [3]. Compared with the cutting of section steel, the groove cutting is complex and diverse, and the requirements for steel cutting equipment are higher. At present, many domestic and foreign cutting equipment on the market range, on the whole, the type of cutting steel foreign steel cutting machine, but the price is expensive [4]; steel cutting machine in the domestic market, mostly for a type of steel cutting, cutting range, according to the $\mathrm{H}$ type steel, channel steel, angle steel and I cut less. Moreover, some cutting equipment needs cutting with manpower, so that the complex groove can not meet the requirements of high efficiency and accuracy. [5-9]. Therefore, in the domestic market, it is necessary to develop a type of steel cutting equipment for cutting $\mathrm{H}$ steel, channel steel, angle steel and i-beam.

\section{Theoretical Analysis}

Kinematics of Robot Manipulator for Cutting Groove

The mathematical model of the manipulator of the cutting groove is established according to the improved D-H method, and the connecting rod coordinate system is shown in Figure1 


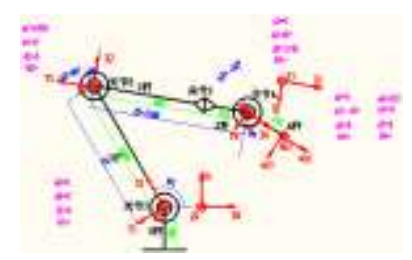

Fig.1 Connecting rod coordinate system for cutting groove manipulator

According to the improved $\mathrm{D}-\mathrm{H}$ method, the pose of the manipulator relative to the base coordinate $\{0\}$ for cutting torch:

$$
\begin{aligned}
& n_{x}=-c_{1}\left(s_{2}\left(c_{4} s_{5}+c_{5} s_{4}\right)-c_{2} c_{3}\left(c_{4} c_{5}-s_{4} s_{5}\right)\right)-s_{1}\left(c_{2}\left(c_{4} s_{5}+s_{4} c_{5}\right)+c_{3} c_{2}\left(c_{4} c_{5}-s_{5} s_{4}\right)\right) \\
& n_{y}=c_{1}\left(c_{2}\left(c_{4} s_{5}+c_{5} s_{4}\right)+s_{2} c_{3}\left(c_{4} c_{5}-s_{4} s_{5}\right)\right)-s_{1}\left(s_{2}\left(c_{4} s_{5}+s_{4} c_{5}\right)-c_{3} c_{2}\left(c_{4} c_{5}-s_{5} s_{4}\right)\right) \\
& n_{z}=s_{3}\left(c_{5} c_{4}-s_{5} s_{4}\right) \\
& o_{x}=-c_{1}\left(s_{2}\left(c_{4} c_{5}-s_{5} s_{4}\right)+c_{2} c_{3}\left(c_{4} s_{5}+s_{4} c_{5}\right)\right)-s_{1}\left(c_{2}\left(c_{4} c_{5}-s_{4} s_{5}\right)-c_{3} c_{2}\left(c_{4} s_{5}+c_{5} s_{4}\right)\right) \\
& o_{y}=c_{1}\left(c_{2}\left(c_{4} c_{5}-s_{5} s_{4}\right)-s_{2} c_{3}\left(c_{4} s_{5}+s_{4} c_{5}\right)\right)-s_{1}\left(s_{2}\left(c_{4} c_{5}-s_{4} s_{5}\right)+c_{3} c_{2}\left(c_{4} c_{5}+c_{5} s_{4}\right)\right) \\
& o_{z}=-s_{3}\left(s_{5} c_{4}+c_{5} s_{4}\right) \\
& a_{x}=s_{1} s_{2} s_{3}-c_{1} c_{2} s_{3} \\
& a_{y}=s_{1} c_{2} s_{3}-c_{1} s_{2} s_{3} \\
& a_{z}=c_{3} \\
& p_{x}=c_{1}\left(310 c_{2} c_{3} c_{4}-s_{2}\left(310 s_{4}-1110\right)+1000\right)-s_{1}\left(c_{2}\left(310 s_{4}-1110\right)+310 c_{3} c_{4} s_{2}\right) \\
& p_{y}=c_{1}\left(c_{2}\left(310 s_{4}-1110\right)+310 s_{2} c_{3} c_{4}\right)+s_{1}\left(310 c_{2} c_{3} c_{4}-s_{2}\left(310 s_{4}-1110\right)+1000\right) \\
& p_{z}=310 c_{4} s_{3} \\
& { }_{5}^{0} T={ }_{1}^{0} T_{5}^{1} T=\left[\begin{array}{cccc}
\mathrm{n}_{\mathrm{x}} & \mathrm{O}_{\mathrm{x}} & \mathrm{a}_{\mathrm{x}} & \mathrm{p}_{\mathrm{x}} \\
\mathrm{n}_{\mathrm{y}} & \mathbf{O}_{\mathrm{y}} & \mathrm{a}_{\mathrm{y}} & \mathrm{p}_{\mathrm{y}} \\
\mathrm{n}_{\mathrm{z}} & \mathrm{O}_{\mathrm{z}} & \mathrm{a}_{\mathrm{z}} & \mathrm{p}_{\mathrm{z}} \\
0 & 0 & 0 & 1
\end{array}\right]
\end{aligned}
$$

The equation of motion of the manipulator is:

$$
\left[\begin{array}{llll}
\mathrm{n}_{x} & \mathrm{o}_{\mathrm{x}} & \mathrm{a}_{\mathrm{x}} & \mathrm{p}_{\mathrm{x}} \\
\mathrm{n}_{\mathrm{y}} & \mathrm{o}_{\mathrm{y}} & \mathrm{a}_{\mathrm{y}} & \mathrm{p}_{\mathrm{y}} \\
\mathrm{n}_{z} & \mathrm{O}_{z} & \mathrm{a}_{z} & \mathrm{p}_{z} \\
0 & 0 & 0 & 1
\end{array}\right]={ }_{1}^{0} T_{2}^{1} T_{3}^{2} T_{3}^{3} T_{4}^{4} T_{5}^{4} T
$$

The matrix elements of the matrix equation is respectively parameter attitude matrix and position vector of the manipulator of a torch, after determining the pose of the torch is known as the matrix of the right side of the equation is unknown, and they depend on the size of the joint angle theta. Invert the left multiply sides, and get the joint rotation:

Kinematic analysis and verification of 3 section steel cutting manipulator model of Robotics steel cutting manipulator. The trajectory planning simulation of the steel cutting manipulator is based on the Robotics Toolbox in MATLAB to construct the 3D model of the steel cutting manipulator. Manipulator model of cutting path "L1 = link" ([0, 100000, 0],'standard') L2 = link ([0, 111000, 0],'standard') L3 = link ([0, 31000, 0],'standard') $\mathrm{R}=\operatorname{robot}(\{\mathrm{L} 1, \mathrm{~L} 2, \mathrm{~L} 3\}$, 'CRobot') Plot (R, [0, 0, 0]) Drivebot $(\mathrm{R})$ Run the above procedures, such as robot 3D model cutting path is shown in Figure 6, $\mathrm{x}, \mathrm{YZ}$, said the torch position manipulator, ax, ay, AZ, Q2, Q1 torch posture, Q3 joint angle control, lower left corner of the map control box can change the slider manipulator's position and attitude .

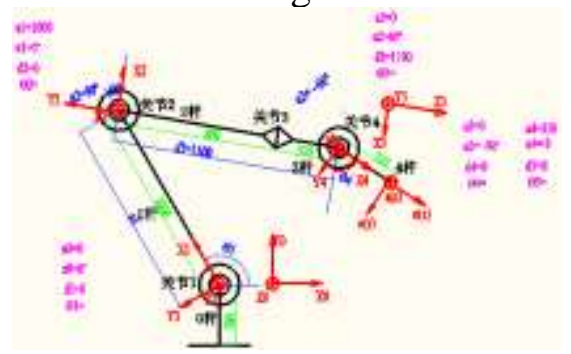

Fig. 2 Connecting rod coordinate system for cutting groove manipulator 
Similarly, a manipulator model for cutting the groove can be built, as shown in Fig. 2 which 3D model of manipulator with cutting groove. It is proved that the slider value of the model steel cutting manipulator is consistent with the kinematics equation MATLAB. Verification of kinematics and inverse kinematics of 3.2 section steel cutting manipulator. The kinematics of the steel cutter manipulator is solved by using the fkine function in MATLAB Robotics Toolbox. Cutting path manipulator kinematics is verified. Suppose $\mathrm{q} 1=[\mathrm{pi} / 2-\mathrm{pi} / 4 \mathrm{pi} / 4]$ (1) solve by MATLAB's fkine function: (2) using positive solution mathematical model 3 to solve: It is proved that the kinematics of the manipulator is correct and the mathematical model is correct. The kinematics of the cutting edge manipulator is verified. Suppose $\mathrm{q} 1=[\mathrm{pi} / 4$ 0, pi/2, -pi/4, 0] (1) solve by MATLAB's fkine function:

$$
\begin{aligned}
& n_{x}=-c_{1}\left(s_{2}\left(c_{4} s_{5}+c_{5} s_{4}\right)-c_{2} c_{3}\left(c_{4} c_{5}-s_{4} s_{5}\right)\right)-s_{1}\left(c_{2}\left(c_{4} s_{5}+s_{4} c_{5}\right)+c_{3} c_{2}\left(c_{4} c_{5}-s_{5} s_{4}\right)\right) \\
& n_{y}=c_{1}\left(c_{2}\left(c_{4} s_{5}+c_{5} s_{4}\right)+s_{2} c_{3}\left(c_{4} c_{5}-s_{4} s_{5}\right)\right)-s_{1}\left(s_{2}\left(c_{4} s_{5}+s_{4} c_{5}\right)-c_{3} c_{2}\left(c_{4} c_{5}-s_{5} s_{4}\right)\right) \\
& n_{z}=s_{3}\left(c_{5} c_{4}-s_{5} s_{4}\right) \\
& o_{x}=-c_{1}\left(s_{2}\left(c_{4} c_{5}-s_{5} s_{4}\right)+c_{2} c_{3}\left(c_{4} s_{5}+s_{4} c_{5}\right)\right)-s_{1}\left(c_{2}\left(c_{4} c_{5}-s_{4} s_{5}\right)-c_{3} c_{2}\left(c_{4} s_{5}+c_{5} s_{4}\right)\right) \\
& o_{y}=c_{1}\left(c_{2}\left(c_{4} c_{5}-s_{5} s_{4}\right)-s_{2} c_{3}\left(c_{4} s_{5}+s_{4} c_{5}\right)\right)-s_{1}\left(s_{2}\left(c_{4} c_{5}-s_{4} s_{5}\right)+c_{3} c_{2}\left(c_{4} c_{5}+c_{5} s_{4}\right)\right) \\
& o_{z}=-s_{3}\left(s_{5} c_{4}+c_{5} s_{4}\right) \\
& a_{x}=s_{1} s_{2} s_{3}-c_{1} c_{2} s_{3} \\
& a_{y}=s_{1} c_{2} s_{3}-c_{1} s_{2} s_{3} \\
& a_{z}=c_{3} \\
& p_{x}=c_{1}\left(310 c_{2} c_{3} c_{4}-s_{2}\left(310 s_{4}-1110\right)+1000\right)-s_{1}\left(c_{2}\left(310 s_{4}-1110\right)+310 c_{3} c_{4} s_{2}\right) \\
& p_{y}=c_{1}\left(c_{2}\left(310 s_{4}-1110\right)+310 s_{2} c_{3} c_{4}\right)+s_{1}\left(310 c_{2} c_{3} c_{4}-s_{2}\left(310 s_{4}-1110\right)+1000\right) \\
& p_{z}=310 c_{4} s_{3}
\end{aligned}
$$

The positive mathematical model 6 to solve::

$$
\theta_{5}=\arcsin \left(\frac{o_{z}}{\mp \sqrt{1-a_{z}^{2}}}\right)-\arccos \frac{p_{z}}{ \pm 310 \sqrt{1-a_{z}^{2}}}
$$

Similarly, it proves that the kinematics of robot manipulator is correct.

\section{Kinematic Analysis and Verification of Steel Cutting Manipulator}

The trajectory planning simulation of steel cutting manipulator is based on the Robotics Toolbox in MATLAB to construct the 3D model which is the steel cutting manipulator. It is can be seen Fig. 3 and Fig.4.

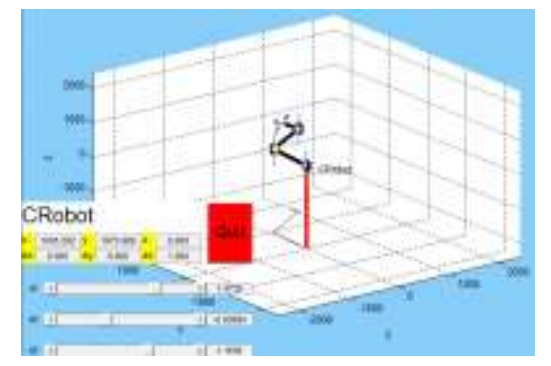

Fig. 3 The model of cutting path manipulator

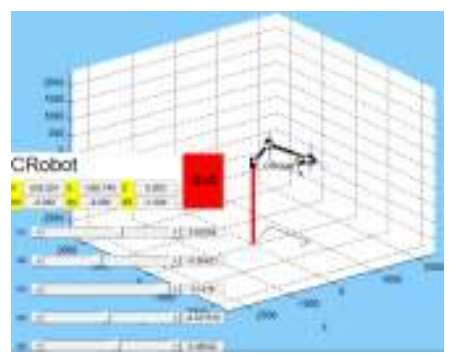

Fig. 4 The model of setting path

The kinematics of the steel cutter manipulator is solved by using the fkine function in MATLAB Robotics Toolbox. The cutting path manipulator kinematics is verified through equation 6.

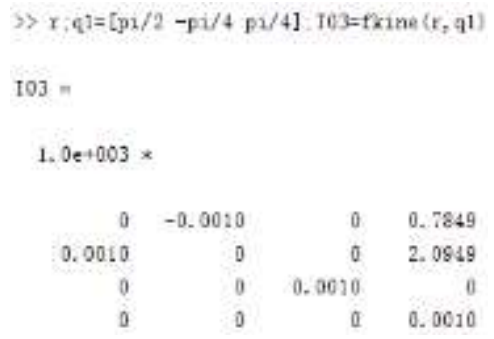

$\begin{array}{rrrr}0 & -0.0010 & 0 & 0.7849 \\ 0.0010 & 0 & 0 & 2.0849 \\ 0 & 0 & 0.0010 & 0 \\ 0 & 0 & 0 & 0.0010\end{array}$


Suppose $\mathrm{q} 1=[\mathrm{pi} / 2 \mathrm{-pi} / 4 \mathrm{pi} / 4]$, Using the inverse mathematical model 5 to solve:

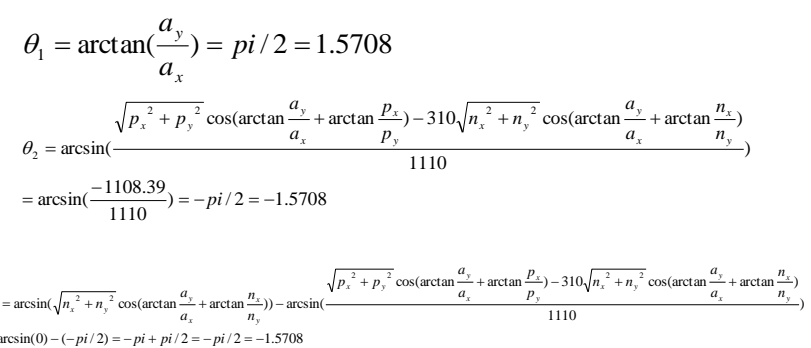

That is theta $1=1.5708$, theta $2=-1.5708$, theta $3=-1.5708$. By using the ikine function in MATLAB and the mathematical model of inverse solution, the result of the solution is consistent. The correctness of the inverse kinematics mathematical model of manipulator is proved. The inverse kinematics of manipulator with groove cutting. Homogeneous transformation matrix corresponding to the manipulator pose hypotheses for cutting torch: (1) the inverse kinematics of the ikine function of MATLAB is used. That is theta $1=0.7854$, theta $2=0$, theta $3=1.5708$, theta $4=-0.7854$, theta $5=0$. (2) using inverse solution mathematical model 8 and 9 to solve: That is theta $1=0.7854$, theta $2=0$, theta $3=1.5708$, theta $4=-0.7854$, theta $5=0$. By using MATLAB's ikine function, kinematic inversion and inverse mathematical model, the results are consistent. It proves the correctness of the mathematical model of kinematics of the manipulator with the cutting groove

\section{Result and Discussion}

This paper describes the design of a common type of steel can be used, and to meet the various needs of the steel cutting process of cutting manipulator, establish the kinematic positive and negative solutions of the mathematical model, and its correctness is verified by MATLAB.

\section{References}

[1] Ma Rupo. Hot rolled medium H type steel rolling process simulation system [D]. East University, 2012:34.

[2] Xing Yongqing. Development and application of CNC cutting machine [J]. technology entrepreneur, 2013,13:73.

[3], Li Shumin, Deng Haitao, Yang Junshu, et al. H steel cutting based on multi robot Numerical control system research [J]. machine tool and hydraulic, 2011,21:98-100.

[4] Zhang Shiliang, Zhang Jian, Lu Jun,.H steel, numerical control flame cutting equipment, mechanical system Design $[\mathrm{J}]$. mechanical and electrical product development and innovation, 2009, v.22; No.11401: 147-149.

[5] The status of cutting machine industry and the development trend of "[J]." during 12th Five-Year Metal processing (hot working), 2011, No.62902:20-22.

[6]Mori, Shozo, H., Nishihara, and, K., Fumta,.Control, Unstable mechanical system:Contro1 of Pendulum[J].Int.J.of, control, 1976,23 (5): 673-692.

[7], Guo Fayong, Mei Tao, Zhao Jianghai,.D-H method to establish the problem of connecting rod coordinate system. Title and improvement $[\mathrm{J}]$. Chinese mechanical engineering, 2014,20:2710-2714.

[8], Jin Long, Jin Jianqiao, Chen Yueping, et al. A kind of three degree of freedom hybrid manipulator Dynamic analysis, [J]. mechanical design and research, 2014,02:19-21.

[9] Jin Long, Jin Jianqiao, Chen Yueping, Qiu Minmin. A hybrid machine with three degrees of freedom Kinematic analysis of arm [J]. mechanical design and research, 2014,02:19-21. 